\title{
CORRELATION BETWEEN SURGICAL LUNG BIOPSY AND AUTOPSY FINDINGS AND CLINICAL DATA IN PATIENTS WITH DIFFUSE PULMONARY INFILTRATES AND ACUTE RESPIRATORY FAILURE
}

\author{
Mauro Canziana, Alexandre de Matos Soeiro ${ }^{\mathrm{b}}$, Marcel Frederico de Lima Taga ${ }^{\mathrm{c}}$, \\ Carmen Silvia Valente Barbas ${ }^{\mathrm{a}}$, Vera Luiza Capelozzi ${ }^{\mathrm{b}}$
}

Canzian M, Soeiro A de M, Taga MF de L, Barbas CSV, Capelozzi VL. Correlation between surgical lung biopsy and autopsy findings and clinical data in patients with diffuse pulmonary infiltrates and acute respiratory failure. CLINICS. 2006(5):425-32.

INTRODUCTION: Surgical lung biopsy is an invasive procedure performed when other procedures have failed to provide an urgent and specific diagnosis, but there may be reluctance to perform it in critically ill patients with diffuse pulmonary infiltrates. PURPOSE: To evaluate the diagnostic accuracy, the changes in therapy and survival of patients with diffuse lung infiltrates, mostly presenting acute respiratory failure, who underwent surgical biopsy.

METHODS: We retrospectively examined medical records and surgical lung biopsies from 1982 to 2003 of 63 patients older than 18 years with diffuse infiltrates. Clinical diagnoses were compared to histopathological ones, from biopsies and autopsies. Laboratory and epidemiological data were evaluated, and their relationship to hospital survival was analyzed.

RESULTS: All histological specimens exhibited abnormalities, mostly presenting benign/inflammatory etiologies. Fifteen patients had an etiologic factor determined in biopsy, most commonly Mycobacterium tuberculosis. The preoperative diagnosis was rectified in 37 patients. Autopsies were obtained in 25 patients and confirmed biopsy results in $72 \%$ of cases. Therapy was changed for $65 \%$ of patients based on biopsy results. Forty-nine percent of patients survived to be discharged from the hospital. Characteristics that differed significantly between survivors and nonsurvivors included sex $(P=0.05)$, presence of comorbidity $(P$ $=0.05), \mathrm{SpO}_{2}(P=0.05)$, and presence of diffuse alveolar damage in the biopsy $(P=0.004)$.

CONCLUSION: Surgical lung biopsy provided a specific, accurate etiologic diagnosis in many patients with diffuse pulmonary infiltrates when clinical improvement did not occur after standard treatment. Surgical lung biopsy may reveal a specific diagnosis that requires distinct treatment, and it would probably have an impact in lowering the mortality of these patients.

KEYWORDS: Surgical lung biopsy. Diffuse pulmonary infiltrate. Acute respiratory failure. Diffuse alveolar damage. Autopsy.

\section{INTRODUCTION}

Surgical lung biopsy (SLB) is an invasive procedure performed to assist in treatment efforts when other

aDivisions of Respiratory Diseases and Pathology, Heart Institute (InCor), São Paulo University Medical School - São Paulo/SP, Brazil.

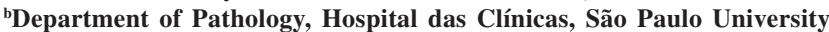
Medical School - São Paulo/SP, Brazil.

'Department of Biostatistics, Federal University of São Paulo/São Paulo/SP, Brazil.

Email: vcapelozzi@lim05.fm.usp.br

Received for publication on June 13, 2006.

Accepted for publication on June 27, 2006. noninvasive or minimally invasive procedures have failed to provide an urgent and specific diagnosis. ${ }^{1,2}$ Surgical lung biopsy is performed through a limited thoracotomy, without costal arc resection, allowing tissue samples to be obtained from 1 or more involved lobe $(\mathrm{s})^{1}$ for adequate histological, histochemical, and molecular analysis. However, there may be reluctance to perform SLB in critically ill patients with diffuse pulmonary infiltrates, who are frequently suffering from acute respiratory failure, often undergoing mechanical ventilation, and hemodynamically unstable. The progression of the disease and the need of a specific 
diagnosis are decisive factors. The purpose of this study was to evaluate diagnostic accuracy, changes in therapy, and survival of patients with diffuse lung infiltrates, mostly presenting acute respiratory failure, who underwent SLB.

\section{METHOD}

Patients. We examined the medical records of all patients who underwent SLB at our institutions from January 1982 to December 2003. From these we selected patients older than 18 years of age with diffuse infiltrates on chest roentgenogram and respiratory failure. Respiratory failure was defined as a $\mathrm{PaO}_{2}$ of less than $60 \mathrm{mmHg}$ (hypoxemic ARF), or a $\mathrm{SpO}_{2}$ less than $90 \%$ of arterial blood, or $\mathrm{PaCO}_{2}$ more than $50 \mathrm{~mm} \mathrm{Hg}$ (hypercapnic acute respiratory failure) while breathing room air. Acute was defined as onset of symptoms of respiratory distress less than 10 days prior to SLB, in order to exclude those patients with a steady deterioration of a chronic process. Surgical lung biopsy was performed at the discretion of the attending physician under general anesthesia; progressive hypoxemia was the usual indication. Through a limited thoracotomy, tissue samples were removed with a cutting stapler. A specific protocol for specimen processing was followed. A portion of the surgical specimen was sent to surgical pathology, and permanent sections were examined after appropriate staining. The remaining specimen was sent to the microbiology laboratory where smears were prepared and part of the tissue was homogenized. Cultures were prepared for aerobic and anaerobic bacteria, fungi, mycobacteria, Legionella, Nocardia, Chlamydia, and viruses. Stains were performed with Gram stain for bacteria, Grocott for fungi and Pneumocystis carinii, and Ziehl-Neelsen for mycobacteria and Nocardia. Results of stains were usually available within 3 hours after specimen arrival in the laboratory.

Statistical Analysis. Comparisons between groups were made using chi-square analysis and unpaired $t$ test when appropriate; $P<0.05$ was taken as significant. Every analysis was performed with the SPSS for Windows 10.0 statistical software.

\section{RESULTS}

Patient Characteristics. Over the period examined, 303 surgical lung biopsies were performed, and 63 cases (20.8\%) met our study criteria. Thirty-one patients (49\%) were women and $32(51 \%)$ were men, with a median age of 51 years (range, 18 to 92 years). Arterial blood samples for gas analysis taken during inspiration of room air in the 24 hours preceding SLB were obtained from 56 patients
(89\%). Forty-four patients were in acute respiratory failure as indicated by the following: 38 of them had a $\mathrm{PaO}_{2}$ $<60 \mathrm{~mm} \mathrm{Hg}$; 4 had a $\mathrm{PaCO}_{2}>50 \mathrm{~mm} \mathrm{Hg}$; and in 2 patients, both $\mathrm{PaO}_{2}$ and $\mathrm{PaCO}_{2}$ indices were altered. Eleven cases had only $\mathrm{SpO}_{2}$ values (under 90\%) available. Tables 1 and 2 list respectively the gasometric data and preexisting medical conditions in the study population. Thirty-three patients (52\%) were immunocompromised, and 48 (66\%) had preexisting medical conditions. The mean time from admission to SLB was 18 days (range, 0 to 61 days), the mean interval from SLB to hospital discharge or death was 21 days (range, 1 to 143 days) and the mean total hospitalization time was 39 days (range, 4 to 184 days).

Diagnostic Results. The diagnostic results of SLB are shown in Table 3. All biopsy specimens exhibited abnormalities. Forty-eight patients (76\%) had benign/inflammatory etiologies; $14 \%$ had infectious causes, and $10 \%$ had infiltrates directly or indirectly due to neoplastic process. Among the main benign/inflammatory etiologies, 26 patients $(41.3 \%)$ had diffuse alveolar damage (DAD) - as the only histopathological diagnosis in 7 patients, or associated with the main process in 19 patients. Twenty one of these were in the exudative stage and 5 in the proliferative stage. Acute lung injury was observed in 6 cases. At least 15 patients received steroid treatment, but in only 3 cases was corticotherapy started after the histopathological result (Table 4). Only 1 patient was in acute respiratory distress clinically. Seven patients (11\%) had usual interstitial pneumonia (UIP), and 7 patients had chronic bronchiolitis, both in acute exacerbation and discordant radiology vs histopathology. Seven patients $(11 \%)$ had a granulomatous process ( 2 sarcoidosis and 5 tuberculosis); 5 patients $(8 \%)$ had pulmonary thromboembolism, and 4 patients $(6.3 \%)$ had passive congestion. For 15 patients (23\%) an etiologic factor was determined through the biopsy as follows: acute and chronic broncho-aspiration in 4 patients $(6.3 \%)$ and infectious etiologic factor in 11 patients $(17 \%)$. The most common cause found was tuberculosis, occurring in 5 patients $(8 \%)$, followed by cytomegalovirus in 2 patients (3\%), Pneumocystis carinii in 2 patients (3\%), Strongyloides $s p$. in 1 patient (1\%), and Aspergillus sp. in 1 patient $(1 \%)$. Clinical, biopsy, and necropsy diagnoses were compared (Table 3). In this situation, the preoperative diagnosis of the cause of respiratory failure or diffuse pulmonary infiltrate was changed in 37 patients (59\%). Autopsies were obtained in 25 patients who died in hospital and confirmed SLB results in 18 cases (72\%). Therapy was changed in 31 patients $(65 \%)$ based on SLB results. Change in therapy was defined as the as the addition or subtraction of 1 or more drugs or the early discontinuation of aggressive life support. Drug changes usually involved anti- 
Table 1 - Gasometric data of the 63 patients studied, previous to surgical lung biopsy

\begin{tabular}{|c|c|c|c|c|c|c|c|c|c|c|c|}
\hline Case & pH & $\mathrm{PaO}_{2}$ & $\mathrm{PaCO}_{2}$ & $\mathrm{SpO}_{2}$ & $\mathrm{Fi} \mathrm{O}_{2}$ & Case & pH & $\mathrm{PaO}_{2}$ & $\mathrm{PaCO}_{2}$ & $\mathrm{SpO}_{2}$ & $\mathrm{Fi} \mathrm{O}_{2}$ \\
\hline 1 & 7,36 & 100 & 30 & 97 & 60 & 33 & 7,48 & 57 & 36 & 92 & NA \\
\hline 2 & 7,08 & 73 & 70 & 90 & 95 & 34 & 7,43 & 44,5 & 44,8 & 82,4 & NA \\
\hline 3 & 7,45 & 33 & 35 & 59,9 & NA & 35 & NA & NA & NA & 87 & NA \\
\hline 4 & 7,31 & 56 & 31 & 85,6 & 60 & 36 & NA & NA & NA & 88 & NA \\
\hline 5 & 7,39 & 66 & 44,9 & 92,9 & NA & 37 & NA & NA & NA & NA & NA \\
\hline 6 & NA & NA & NA & 80 & NA & 38 & 7,42 & 30,9 & 28,4 & 59,6 & NA \\
\hline 7 & 7,38 & 51,8 & 42 & 85 & NA & 39 & 7,28 & 48,1 & 29 & 76,2 & NA \\
\hline 8 & NA & NA & NA & 88 & NA & 40 & NA & NA & NA & 96 & 60 \\
\hline 9 & 7,36 & 120 & 36 & 96,3 & 60 & 41 & 7,42 & 50,6 & 34,7 & 86,2 & 50 \\
\hline 10 & 7,43 & NA & 50,1 & 86,9 & 100 & 42 & NA & NA & NA & NA & NA \\
\hline 11 & 7,46 & 51 & 28,6 & 86 & NA & 43 & 7,42 & 52 & 25 & 88,3 & 60 \\
\hline 12 & 7,22 & 57,9 & 62,4 & 88,2 & 45 & 44 & 7,43 & 51,1 & 36,8 & 84 & NA \\
\hline 13 & 7,39 & 58,2 & 40,3 & 89,6 & NA & 45 & 7,46 & 59,5 & 37 & 92 & NA \\
\hline 14 & 7,43 & 125,9 & 19,5 & 98,8 & 40 & 46 & 7,33 & 47 & 46,5 & 79,5 & NA \\
\hline 15 & 7,44 & 45,3 & 27,5 & 82,5 & NA & 47 & 7,03 & 49 & 87 & 63,1 & 40 \\
\hline 16 & 7,5 & 46,3 & 29 & 86,7 & 100 & 48 & NA & NA & NA & 90 & NA \\
\hline 17 & 7,35 & 29 & 40 & 49,1 & NA & 49 & 7,13 & 65 & 63 & 85,8 & 100 \\
\hline 18 & NA & NA & NA & NA & NA & 50 & NA & NA & NA & 96 & NA \\
\hline 19 & NA & NA & NA & 87 & NA & 51 & NA & NA & NA & NA & NA \\
\hline 20 & 7,51 & 48,3 & 26,3 & 88,4 & 60 & 52 & 7,37 & 42,8 & 23,5 & 75,7 & NA \\
\hline 21 & 7,56 & 67 & 25 & 95,6 & NA & 53 & 7,47 & 59,8 & 29,6 & 92,4 & NA \\
\hline 22 & NA & NA & NA & 83 & NA & 54 & 7,36 & 40 & 46 & 72 & NA \\
\hline 23 & 7,37 & 54 & 40,7 & 86,6 & NA & 55 & NA & NA & NA & NA & NA \\
\hline 24 & NA & NA & NA & 68 & 100 & 56 & 7,44 & 38,9 & 33,8 & 75,8 & 50 \\
\hline 25 & 7,42 & 80 & 27 & 96,2 & NA & 57 & NA & NA & NA & 80 & NA \\
\hline 26 & 7,48 & 43 & 29 & 80 & 60 & 58 & NA & NA & NA & NA & NA \\
\hline 27 & NA & NA & NA & NA & NA & 59 & 7,49 & 87,1 & 23,9 & 97,3 & NA \\
\hline 28 & 7,4 & 27,2 & 37,2 & 49,9 & 80 & 60 & 7,49 & 45,2 & 37,2 & 85 & NA \\
\hline 29 & 7,43 & 71,7 & 32,1 & 94,6 & NA & 61 & 7,1 & 79 & 54 & 88 & 40 \\
\hline 30 & 7,41 & 36,5 & 42,5 & 70,1 & NA & 62 & 7,23 & 105,9 & 20,4 & 96,3 & 40 \\
\hline 31 & NA & 41,5 & 33 & 77,5 & NA & 63 & 7,43 & 52,2 & 38 & 87,8 & NA \\
\hline 32 & 7,44 & 35,5 & 38 & 70,8 & 40 & & & & & & \\
\hline
\end{tabular}

NA: data non-available

Note: Data concerning $\mathrm{PaO}_{2}$ and $\mathrm{PaCO}_{2}$ expressed in $\mathrm{mmHg}$; data concerning $\mathrm{SpO}_{2}$ an $\mathrm{FiO}_{2}$ expressed in percentage.

biotics and/or corticosteroids, but also included heparinization and the initiation or discontinuation of antineoplastic agents for malignancy or drug-induced toxicity, respectively. Surgical complication data was obtained in 32 patients; surgical complications were present in 5 patients (1 infection of the surgical wound, 1 pneumothorax, and 3 subcutaneous emphysema, Table 4).

Survival. Thirty-one patients (49\%) survived to be discharged from the hospital.. For the 34 patients who died in hospital, survival time after SLB was $20 \pm 29$ days. Although not statistically significant, alterations in therapy resulting from SLB modified the survival rate in 7 patients $(22 \%)$ out of 32 patients who had the information available $\left(X^{2}=0.14, P=0.4\right)$. A marginal significance was found between mortality and the presence of surgical complications $\left(X^{2}=3.80, P=0.05\right)$. In addition, neither the availability of a specific diagnosis nor changes in diagnosis, as well as infectious agent or immunocompromised status affected survival rates $\left(X^{2}=0.44, P=0.24 ; X^{2}=0.51\right.$, $P=0.27$; and $X^{2}=0.13, P=0.08$, respectively).

Preoperative characteristics that did not differ between survivors and nonsurvivors included age $\left(X^{2}=2.42 ; P=\right.$ 0.9.), $\mathrm{PaO}_{2},\left(X^{2}=5.07 ; P=0.1\right) \mathrm{PCO}_{2},\left(X^{2}=4.02 ; P=\right.$ $0.1)$ and breathing frequency at the time of SLB $\left(\left(X^{2}=\right.\right.$ $1.37 ; P=0.6)$. There was no difference between the preoperative primary diagnosis and the diagnosis found at SLB in survivors versus nonsurvivors. Characteristics that differed significantly between survivors and nonsurvivors included sex $\left(X^{2}=3.56 ; P=0.05\right)$, comorbidity $\left(X^{2}=3.56\right.$ ; $P=0.05), \mathrm{SpO}_{2}\left(X^{2}=3.98 ; P=0.05\right)$, and presence of DAD in the SLB $\left(X^{2}=8.38 ; P=0.004\right)$. Eight out of 26 patients $(31 \%)$ with DAD and only 3 out of 11 patients (27 $\%$ ) with infectious etiology survived, in contrast to the case with acute exacerbation of UIP, in which 4 of 7 patients $(57 \%)$ survived. A marginal significance was obtained for the clinical and gasometric pattern of acute respiratory failure $\left(X^{2}=3.1 ; P=0.07\right)$

\section{DISCUSSION}

Although SLB did provide a specific diagnosis in many patients with diffuse pulmonary infiltrates and acute res- 
Table 2 - Epidemiological data of the 63 patients studied

\begin{tabular}{|c|c|c|c|c|c|c|}
\hline Case & Sex & Age & $\begin{array}{l}\text { Total Hospitalization } \\
\text { Time (days) }\end{array}$ & Underlying disease & $\begin{array}{c}\text { Presence of } \\
\text { immunossupression factor }\end{array}$ & Outcome \\
\hline 1 & $\mathrm{~m}$ & 56 & 20 & No & No & Dead \\
\hline 2 & $\mathrm{~m}$ & 48 & 32 & No & No & Dead \\
\hline 3 & $\mathrm{~m}$ & 76 & 60 & Renal and heart failure & No & Survived \\
\hline 4 & $\mathrm{f}$ & 19 & 47 & BMT (aplastic anemia) & Yes & Dead \\
\hline 5 & $\mathrm{~m}$ & 67 & 44 & No & No & Survived \\
\hline 6 & $\mathrm{~m}$ & 55 & 17 & Rheumatologic disease NOS & No & Dead \\
\hline 7 & $\mathrm{f}$ & 52 & 27 & Heart failure & No & Survived \\
\hline 8 & $\mathrm{~m}$ & 48 & 55 & Crohn's disease+ renal failure & No & Dead \\
\hline 9 & $\mathrm{~m}$ & 66 & 42 & Rheumatoid arthritis & No & Dead \\
\hline 10 & $\mathrm{f}$ & 37 & 44 & SLE & No & Dead \\
\hline 11 & $\mathrm{~m}$ & 34 & 16 & AIDS & Yes & Dead \\
\hline 12 & $\mathrm{~m}$ & 45 & 18 & CML & Yes & Dead \\
\hline 13 & $\mathrm{~m}$ & 52 & 20 & No & No & Survived \\
\hline 14 & $\mathrm{f}$ & 21 & 38 & Non-Hodgkin's lymphoma & Yes & Survived \\
\hline 15 & $\mathrm{f}$ & 30 & 37 & No & No & Survived \\
\hline 16 & $\mathrm{f}$ & 63 & 9 & Carcinomatosis & Yes & Dead \\
\hline 17 & $\mathrm{~m}$ & 37 & 48 & No & No & Dead \\
\hline 18 & $\mathrm{f}$ & 51 & 22 & Gastroesophageal reflux & No & Survived \\
\hline 19 & $\mathrm{~m}$ & 52 & 38 & Liver cirrhosis & No & Survived \\
\hline 20 & $\mathrm{f}$ & 46 & 26 & Adenocarcinoma NOS & No & Dead \\
\hline 21 & $\mathrm{f}$ & 34 & 4 & CML & Yes & Dead \\
\hline 22 & $\mathrm{f}$ & 49 & 19 & Carcinomatosis & Yes & Survived \\
\hline 23 & $\mathrm{~m}$ & 23 & 21 & Burns & No & Dead \\
\hline 24 & $\mathrm{~m}$ & 58 & 13 & Adenocarcinoma NOS & Yes & Dead \\
\hline 25 & $\mathrm{~m}$ & 77 & 45 & COPD & Yes & Dead \\
\hline 26 & $\mathrm{~m}$ & 36 & 20 & BMT & Yes & Dead \\
\hline 27 & $\mathrm{~m}$ & 78 & 17 & No & Yes & Survived \\
\hline 28 & $\mathrm{f}$ & 54 & 9 & SLE & No & Dead \\
\hline 29 & $\mathrm{f}$ & 51 & 184 & Wegener's granumatosis & No & Dead \\
\hline 30 & $\mathrm{~m}$ & 57 & 33 & Ischemic myocardiopathy & No & Dead \\
\hline 31 & $\mathrm{f}$ & 21 & 42 & SLE & No & Survived \\
\hline 32 & $\mathrm{f}$ & 74 & 16 & Congestive myocardiopathy & No & Dead \\
\hline 33 & $\mathrm{~m}$ & 55 & 91 & Congestive myocardiopathy & No & Dead \\
\hline 34 & $\mathrm{~m}$ & 65 & 69 & Sarcoidosis & No & Dead \\
\hline 35 & $\mathrm{f}$ & 46 & 15 & Wegener's granumatosis & No & Survived \\
\hline 36 & $\mathrm{f}$ & 52 & 76 & No & Yes & Survived \\
\hline 37 & $\mathrm{f}$ & 92 & 24 & Ischemic myocardiopathy & No & Survived \\
\hline 38 & $\mathrm{f}$ & 26 & 27 & BMT (CML) & Yes & Dead \\
\hline 39 & $\mathrm{f}$ & 53 & 98 & Rheumatoid arthritis & No & Dead \\
\hline 40 & $\mathrm{~m}$ & 70 & 47 & Non-Hodgkin's lymphoma & Yes & Dead \\
\hline 41 & $\mathrm{f}$ & 30 & 65 & Colitis NOS & No & Survived \\
\hline 42 & $\mathrm{f}$ & 82 & 22 & Neoplasia NOS & Yes & Survived \\
\hline 43 & $\mathrm{f}$ & 50 & 91 & SLE & Yes & Survived \\
\hline 44 & $\mathrm{~m}$ & 57 & 38 & Gastroesophageal reflux+DM 2 & No & Survived \\
\hline 45 & $\mathrm{~m}$ & 65 & 37 & Ischemic myocardiopathy+DM 2 & No & Dead \\
\hline 46 & $\mathrm{~m}$ & 47 & 37 & ALL & Yes & Dead \\
\hline 47 & $\mathrm{~m}$ & 32 & 16 & ALL & Yes & Dead \\
\hline 48 & $\mathrm{f}$ & 68 & 37 & Hypertension & No & Survived \\
\hline 49 & $\mathrm{~m}$ & 53 & 26 & Heart transplantation & Yes & Dead \\
\hline 50 & $\mathrm{~m}$ & 22 & 89 & ALL & Yes & Survived \\
\hline 51 & $\mathrm{~m}$ & 18 & 7 & BMT (ALL) & Yes & Survived \\
\hline 52 & $\mathrm{f}$ & 76 & 36 & Ischemic myocardiopathy & No & Dead \\
\hline 53 & $\mathrm{f}$ & 55 & 17 & Carcinomatosis & Yes & Dead \\
\hline 54 & $\mathrm{f}$ & 25 & 47 & No & No & Survived \\
\hline 55 & $\mathrm{f}$ & 38 & 42 & No & No & Dead \\
\hline 56 & $\mathrm{~m}$ & 37 & 84 & CML & Yes & Dead \\
\hline 57 & $\mathrm{f}$ & 36 & 10 & Ischemic myocardiopathy & No & Survived \\
\hline 58 & $\mathrm{f}$ & 36 & 14 & Carcinomatosis & Yes & Survived \\
\hline 59 & $\mathrm{~m}$ & 73 & 34 & No & No & Survived \\
\hline 60 & $\mathrm{f}$ & 52 & 36 & No & No & Survived \\
\hline 61 & $\mathrm{~m}$ & 31 & 49 & SLE & No & Dead \\
\hline 62 & $\mathrm{~m}$ & 28 & 48 & Aplastic anemia & Yes & Dead \\
\hline 63 & $\mathrm{f}$ & 73 & 37 & No & No & Survived \\
\hline
\end{tabular}

Abbreviations: AIDS: Acquired Immunodeficiency Syndrome; ALL: Acute Lymphocytic Leukemia; BMT: Bone Marrow Transplantation; CML: Chronic Myelocytic Leukemia; CPOD: Chronic Obstructive Lung Disease; DM: Diabetes Mellitus; NOS: no other specification; SLE: Systemic Lupus Erythematous 
Table 3 - Distribution of clinical, biopsy and necropsy diagnoses

\begin{tabular}{lcccccc}
\hline Diagnoses & \multicolumn{2}{c}{ Clinical } & \multicolumn{2}{c}{ Biopsy } & \multicolumn{2}{c}{ Necropsy } \\
\hline $\begin{array}{l}\text { Infectious lung } \\
\text { disease }\end{array}$ & 22 & $(35)$ & 9 & $(14)$ & 9 & $(38)$ \\
$\begin{array}{l}\text { Granulomatous } \\
\text { lung disease }\end{array}$ & 7 & $(11)$ & 7 & $(11)$ & 1 & $(4)$ \\
$\begin{array}{l}\text { Airway diseases } \\
\text { Interstitial pneumonia }\end{array}$ & 11 & $(6)$ & 10 & $(16)$ & 1 & $(4)$ \\
$\begin{array}{l}\text { Circulatory disturbance } \\
\text { Carcinoma }\end{array}$ & 6 & $(10)$ & 7 & $(16)$ & 1 & $(4)$ \\
$\begin{array}{l}\text { Hypersensitivity } \\
\text { pneumonia }\end{array}$ & 6 & $(10)$ & 5 & $(8)$ & 3 & $(13)$ \\
$\begin{array}{l}\text { Diffuse alveolar } \\
\text { damage }\end{array}$ & & & 2 & $(3)$ & & \\
$\begin{array}{l}\text { Capilaritis/ vasculitis } \\
\text { Amyloidosis }\end{array}$ & 5 & $(8)$ & 4 & $(6)$ & 1 & $(4)$ \\
$\begin{array}{l}\text { Histiocytosis X } \\
\text { Lymphoma }\end{array}$ & 1 & $(2)$ & 1 & $(2)$ & & \\
Total & 1 & $(2)$ & 1 & $(2)$ & & \\
\hline
\end{tabular}

piratory failure, the hospital mortality rate of $51 \%$ was high in this hypoxic patient population. Hospital mortality rates from similar but smaller series of hypoxic patients undergoing SLB are consistent with our experience, ranging from $50 \%$ to $70 \% .^{3-7}$ In contrast, reported mortality rates for all patients (hypoxic and nonhypoxic) undergoing SLB tend to be lower. ${ }^{4,-10}$ Greenman and coworkers ${ }^{11}$ found a $73 \%$ mortality rate in hypoxic $\left(\mathrm{PaO}_{2}<55 \mathrm{~mm} \mathrm{Hg}\right)$ immunosuppressed patients and a $39 \%$ mortality rate in nonhypoxic patients. In immunosuppressed patients, Jaffe and $\mathrm{Maki}^{12}$ found a significantly higher $\mathrm{PaO}_{2}$ in survivors of SLB, as compared to nonsurvivors. While our study was not designed to evaluate hypoxia as a predictor of mortality in patients undergoing SLB, probably our hypoxic patients ultimately did not fare well.

Given the high mortality rate, it would be helpful to be able to predict outcome. Poe and coworkers ${ }^{13}$ in a retrospective review attempted to predict mortality in immunocompromised patients with pulmonary infiltrates undergoing either transbronchial biopsy or SLB. They found that early mechanical ventilation (within the first 72 hours of admission, either preoperatively or for more than 24 hours postoperatively), the initial room-air $\mathrm{PaO}_{2}$, and corticosteroid therapy at the onset of symptoms were significant predictors of mortality. None of the 51 patients who simultaneously had a $\mathrm{PaO}_{2}$ of $<50 \mathrm{~mm} \mathrm{Hg}$ on room air, was receiving corticosteroids, and required early mechanical ventilation survived.

Most investigators feel that mortality in patients undergoing SLB is due to the severity of the underlying disease and not to complications of the surgical procedure. ${ }^{3,4,14-16}$ With the exception of a mortality percentage of $21.4 \%$ re- ported by Ferson et al, ${ }^{17}$ more recent series refer indices lower than $8 \%$ directly related to the biopsy procedure. ${ }^{18-}$ ${ }^{21}$ While this assertion is difficult to prove, the complications in our series did not appear to directly cause death. However, the mortality rate in the patients suffering complications, while marginally significant, suggests that such complications requires more complex patient care. Studies evaluating the impact of SLB complications on outcome should include a nonoperative control group. In a prospective, randomized study comparing SLB and empiric antibiotic therapy for acute pneumonitis in 22 non-neutropenic cancer patients, Potter and coworkers ${ }^{22}$ did not find a significant difference in mortality rates between the 2 groups. The investigators did feel that 3 patients $(21 \%$ of those undergoing SLB) experienced complications of SLB that contributed directly to their deaths. Although our patients did not suffer this high rate of catastrophic complications, any complication of a procedure of uncertain benefit is of concern.

Intrathoracic surgery, especially in the hypoxic patient, carries with it the risk of prolonged postoperative mechanical ventilation. Although it became possible to extubate half of our patients postoperatively, 6 patients $(50 \%)$ were mechanically ventilated until death. It is not known how many of these patients would have eventually required ventilatory support had SLB not been performed, although only 1 of the patients presented a serious complication (air leak) related to the biopsy.

In the last decades, advances in technology such as computerized tomography, acquisition of knowledge in lung pathology produced by indications of surgical lung biopsy, the new ATS/ERS Classification of the Interstitial Lung Diseases may be an explanation for our $100 \%$ of specificity in diagnosis, in contrast to those found in previous series of SLB in immunosuppressed patients, ranging from $40 \%$ to $83 \% .^{11,12,23-27}$ Autopsy results in 18 (72\%) patients verified the accuracy of SLB in all but 7 cases; the additional diagnosis (4 infectious pneumonias, 5 diffuse alveolar damages, and 1 bronchiolitis) may represent infections or complications resulting from them, which occurred between SLB and death. In many patients, the preoperative diagnosis was either erroneous or incomplete. The impact of SLB on changes in diagnoses, the presence of a specific diagnosis, and subsequent alterations in therapy on survival were very encouraging, and mirrored the experience of many others investigators. ${ }^{71,14,16,28,29}$ Delays in diagnosis can account for the high mortality rate reported in the literature, ${ }^{25,28,30}$ because the study population included many patients undergoing SLB relatively late in the course of their acute respiratory failure. Mortality might have been even greater in the diagnostic group, had a diagnosis not been 
Table 4 - Data concerning complications related to biopsy procedure, change in therapy after histopathological results and corticotherapy before and after histopathological results

\begin{tabular}{|c|c|c|c|c|c|}
\hline Case & Complications & Diffuse alveolar damage & Change in therapy & $\begin{array}{l}\text { Corticotherapy } \\
\text { pre-biopsy }\end{array}$ & $\begin{array}{c}\text { Corticotherapy } \\
\text { post-biopsy }\end{array}$ \\
\hline 1 & no & yes (exs) & yes & yes & yes \\
\hline 2 & no & yes (exs) & yes & no & no \\
\hline 3 & no & yes (pro) & yes & no & no \\
\hline 4 & NA & no & NA & NA & NA \\
\hline 5 & no & no & NA & yes & NA \\
\hline 6 & no & yes (exs) & no\# & no & no \\
\hline 7 & no & no & yes & no & yes \\
\hline 8 & no & yes (exs) & yes & no & no \\
\hline 9 & yes* & no & yes & yes & yes \\
\hline 10 & no & yes (pro) & yes & yes & yes \\
\hline 11 & no & yes (exs) & yes & yes & yes \\
\hline 12 & no & yes (exs) & yes & no & no \\
\hline 13 & NA & no & NA & NA & NA \\
\hline 14 & yes** & no & yes & yes & yes \\
\hline 15 & no & no & yes & no & no \\
\hline 16 & NA & no & NA & NA & NA \\
\hline 17 & no & no & yes & no & no \\
\hline 18 & NA & yes (exs) & NA & NA & NA \\
\hline 19 & no & yes (exs) & yes & no & yes \\
\hline 20 & NA & yes (exs) & NA & NA & NA \\
\hline 21 & no & no & no\# & no & NA \\
\hline 22 & no & yes (pro) & yes & no & no \\
\hline 23 & no & no & no & no & no \\
\hline 24 & NA & yes (exs) & NA & NA & NA \\
\hline 25 & no & no & yes & no & no \\
\hline 26 & NA & yes (exs) & NA & NA & NA \\
\hline 27 & NA & no & NA & NA & NA \\
\hline 28 & no & yes (exs) & no\# & yes & yes \\
\hline 29 & no & no & yes & no & yes \\
\hline 30 & no & no & No & no & no \\
\hline 31 & NA & no & NA & NA & NA \\
\hline 32 & no & yes (pro) & no\# & yes & yes \\
\hline 33 & no & no & No & no & no \\
\hline 34 & no & no & yes & no & no \\
\hline 35 & NA & no & NA & NA & NA \\
\hline 36 & NA & no & NA & NA & NA \\
\hline 37 & NA & no & NA & NA & NA \\
\hline 38 & NA & yes (pro) & NA & NA & NA \\
\hline 39 & no & no & yes & yes & no \\
\hline 40 & yes*** & yes (exs) & No & yes & yes \\
\hline 41 & NA & no & NA & NA & NA \\
\hline 42 & no & no & No & yes & yes \\
\hline 43 & NA & no & NA & NA & NA \\
\hline 44 & NA & no & NA & NA & NA \\
\hline 45 & NA & yes (exs) & NA & NA & NA \\
\hline 46 & NA & no & NA & NA & NA \\
\hline 47 & NA & no & NA & NA & NA \\
\hline 48 & yes*** & yes (exs) & No & yes & yes \\
\hline 49 & no & yes (exs) & Yes & yes & yes \\
\hline 50 & yes*** & no & No & no & no \\
\hline 51 & NA & no & NA & NA & NA \\
\hline 52 & NA & no & NA & NA & NA \\
\hline 53 & NA & no & NA & NA & NA \\
\hline 54 & no & yes (exs) & No & no & no \\
\hline 55 & NA & yes (exs) & NA & NA & NA \\
\hline 56 & NA & yes (exs) & NA & NA & NA \\
\hline 57 & NA & no & NA & NA & NA \\
\hline 58 & NA & no & NA & NA & NA \\
\hline 59 & NA & yes (exs) & NA & NA & NA \\
\hline 60 & NA & no & NA & NA & NA \\
\hline 61 & NA & no & NA & NA & NA \\
\hline 62 & NA & no & NA & NA & NA \\
\hline 63 & NA & yes (exs) & NA & NA & NA \\
\hline
\end{tabular}

NA: data non-available; * Infection of surgical wound; ** Atelectasia and pneumothorax; *** Subcutaneous emphysema; \# Death before histopathological conclusion; "exs": exsudative phase; "pro": proliferative phase 
made, this being a speculation that can be evaluated using our data. Also, patients without a specific diagnosis may have had less severe disease and may have thus been preselected for lower mortality. Nevertheless, while SLB is undoubtedly of value in securing or confirming diagnoses, our results suggest that SLB may influence patient survival rates through the direction of treatment strategy.

If patient benefit is defined by survival, the 6 patients ( $50 \%$ of total) who both survived and in whom SLB re- sults were judged to be useful in guiding therapy can definitely be described as having benefited from SLB.

In conclusion, SLB provided a specific, accurate etiologic diagnosis in many patients with diffuse pulmonary infiltrates and acute respiratory failure when clinical improvement did not occur after standard treatment. Surgical lung biopsy may produce a specific diagnosis that indicates a distinct treatment, lowering patient mortality.

\section{RESUMO}

Canzian M, Soeiro A de M, Taga MF de L, Barbas CSV, Capelozzi VL. Correlação entre achados de biópsia pulmonar cirúrgica e de autópsia e dados clínicos em pacientes com infiltrados pulmonares difusos e insuficiência respiratória aguda. CLINICS. 2006(5):425-32.

INTRODUÇÃO: A biópsia pulmonar cirúrgica é um procedimento invasivo executado quando outros procedimentos não forneceram um diagnóstico urgente e específico; no entanto, pode haver relutância em sua execução em pacientes críticos, que apresentam infiltrados pulmonares difusos.

OBJETIVO: Avaliar a acurácia diagnóstica, mudanças na terapêutica e a sobrevida de pacientes com infiltrado pulmonar difuso, em sua maior parte apresentando a insuficiência respiratória aguda, submetidos a biópsia cirúrgica.

MÉTODO: Foram examinados retrospectivamente registros médicos e biópsias pulmonares cirúrgicas de 63 pacientes maiores de 18 anos de idade, com infiltrados difusos, entre 1982 a 2003. Os diagnósticos clínicos foram comparados aos histopatológicos, de biópsias e de autópsias. Dados laboratoriais e epidemiológicos foram avaliados e sua correlação com a sobrevida hospitalar analisada.

RESULTADOS: Todos os espécimes histológicos exibiram alterações, em sua maior parte de natureza benigna/infla- matória. Em quinze casos o fator etiológico pôde ser determinado na biópsia, sendo o Mycobacterium tuberculosis o mais freqüiente. O diagnóstico pré-operatório foi mudado em 37 pacientes. Autópsias foram realizadas em 25 pacientes e confirmaram resultados da biópsia em $72 \%$. A terapêutica foi alterada em $65 \%$ dos pacientes com base nos resultados da biópsia. Quarenta e nove por cento dos pacientes sobreviveram ao final do período de hospitalização. Características que diferiram significativamente entre sobreviventes versus não sobreviventes incluíram sexo $(\mathrm{p}=0.05)$, a presença de comorbidade $(\mathrm{p}=0.05), \mathrm{a} \mathrm{SatO}_{2}$ $(\mathrm{p}=0.05)$, e a presença de dano alveolar difuso na biópsia $(\mathrm{p}=0.004)$.

CONCLUSÃO: A biópsia pulmonar cirúrgica forneceu um diagnóstico etiológico específico e exato em muitos pacientes com infiltrados pulmonares difusos quando a melhora clínica não ocorreu após o tratamento padrão. A biópsia pulmonar cirúrgica pode fornecer diagnósticos que requerem tratamentos específicos, com provável impacto na redução do índice de mortalidade destes pacientes.

UNITERMOS: Biópsia pulmonar cirúrgica. Infiltrado pulmonar difuso. Insuficiência respiratória aguda. Dano alveolar difuso. Autópsia.

\section{REFERENCES}

1. Monteiro AS, Addor G. Open lung biopsy in patients on mechanical ventilation and presenting diffuse pulmonary infiltrate. J Bras Pneumol. $2005 ; 31: 212-8$

2. Oliveira RHR, Basille Filho A. Incidence of acute lung injury and acute respiratory distress syndrome in the intensive care unit of a university hospital: a prospective study. J Bras Pneumol. 2006;32:35-42

3. Warner DO, Warner MA, Divertie MB. Open lung biopsy in patients with diffuse pulmonary infiltrates and acute respiratory failure. Am Rev Respir Dis. 1988;137:90-4.
4. Wagner JD, Stahler C, Knox S, Brinton M, Knecht B. Clinical utility of open lung biopsy for undiagnosed pulmonary infiltrates. Am J Surg. 1992;164:104-7.

5. Papazian L, Thomas P, Bregeon F, Garbe L, Zandotti C, Saux P, et al. Open-lung biopsy in patients with acute respiratory distress syndrome. Anesthesiology. 1998;88:935-44.

6. Flabouris A, Myburgh J. The utility of open lung biopsy in patients requiring mechanical ventilation. Chest. 1999;115:811-7. 
7. Patel SR, Karmpaliotis D, Ayas NT, Mark EJ, Wain J, Thompson T, et al. The role of open-lung biopsy in ARDS. Chest. 2004;125:197-202.

8. Bove P, Ranger W, Pursel S, Glover J, Bove K, Bendick P. Evaluation of outcome following open lung biopsy. Am Surg. 1994;60:564-70.

9. Kramer MR; Berkman, N; Mintz, B, Godfrey S, Saute M, Amir G. The role of open lung biopsy in the management and outcome of patients with diffuse lung disease. Ann Thorac Surg. 1998;65:198-202.

10. Bousso A, Baldacci ER, Fernandes JC, Fernandes ICO, Cordeiro AMG, Otoch JP, et al. Contribuição da biópsia pulmonar cirúrgica na avaliação de pneumopatias difusas e agudas em unidade de terapia intensiva pediátrica. J Pneumol. 1987;28:30-40.

11. Greenman RL, Goodall PT, King D. Lung biopsies in immunocompromised hosts. Am J Med. 1975;59:488-96.

12. Jaffe JP, Maki DG. Lung biopsy in immunocompromised patients: one institution's experience and an approach to management of pulmonary disease in the compromised host. Cancer. 1981;48:1144-53.

13. Poe RH, Wahl GW, Qazi R, Kallay MC, Utell MJ, Morrow GR. Predictors of mortality in the immunocompromised patient with pulmonary infiltrates. Arch Intern Med. 1986;146:1304-8.

14. Walker WA, Cole FH Jr, Khandekar A, Mahfood SS, Watson DC. Does open lung biopsy affect treatment in patients with diffuse pulmonary infiltrates? J Thorac Cardiovasc Surg. 1989;97:534-40.

15. Shah SS, Tsang V, Goldstraw P. Open lung biopsy: a safe, reliable and accurate method for diagnosis in diffuse lung disease. Respiration. 1992;59:243-6.

16. Neuhaus SJ, Mattar KS. The efficacy of open lung biopsy. Aust N Z J Surg. 1997;67:181-4.

17. Ferson PF, Landreneau RJ, Dowling RD, Hazelrigg SR, Ritter P, Nunchuck $\mathrm{S}$, et al. Comparison of open versus thoracoscopic lung biopsy for diffuse infiltrative pulmonary disease. J Thorac Cardiovasc Surg. 1993;106:194-9.

18. Temes RT, Joste NE, Qualls CR, Allen NL, Crowell RE, Dox HA, et al. Lung biopsy: is it necessary? J Thorac Cardiovasc Surg. 1999;118:1097100 .

19. Ayed AK, Raghunathan R. Thoracoscopy versus open lung biopsy in the diagnosis of interstitial lung disease: a randomised controlled trial. J R Coll Surg Edinb. 2000;45:159-163.
20. Lettieri CJ, Veerappan GR, Helman DL, Mulligan CR, Shorr AF Outcomes and safety of surgical lung biopsy for interstitial lung disease. Chest. 2005;127:1600-5.

21. Lee YC, Wu CT, Hsu HH, Huang PM, Chang YL. Surgical lung biopsy for diffuse pulmonary disease: experience of 196 patients. J Thorac Cardiovasc Surg. 2005;129:984-90.

22. Potter D, Pass HI, Brower S, Macher A, Browne M, Thaler, M, et al Prospective randomized study of open lung biopsy versus empirical antibiotic therapy for acute pneumonitis in nonneutropenic cancer patients. Ann Thorac Surg; 1985;40:422-8.

23. Leight GS Jr, Michaelis LL. Open lung biopsy for the diagnosis of acute, diffuse pulmonary infiltrates in the immunosuppressed patient. Chest. 1978;73:477-82.

24. Haverkos HW, Dowling JN, Pasculle AW, Myerowitz RL, Lerberg DB, Hakala TR. Diagnosis of pneumonitis in immunocompromised patients by open lung biopsy. Cancer. 1983;52:1093-7.

25. Cheson BD, Samlowski WE, Tang TT, Spruance SL. Value of openlung biopsy in 87 immunocompromised patients with pulmonary infiltrates. Cancer. 1985;55:453-9.

26. McCabe RE, Brooks RG, Mark JB, Remington JS. Open lung biopsy in patients with acute leukemia. Am J Med. 1985;78:609-16.

27. Thomas JH, Farek PE, Hermreck AS, Pierce GE. Diagnostic value of open lung biopsy in immunocompromised patients. Am J Surg. 1987,154:692-5.

28. Rossiter SJ, Miller C, Churg AM, Carrington CB, Mark JB. Open lung biopsy in the immunosuppressed patient. Is it really beneficial? J Thorac Cardiovasc Surg. 1979;77:338-45.

29. Canver CC, Mentzer RM Jr. The role of open lung biopsy in early and late survival of ventilator-dependent patients with diffuse idiopathic lung disease. J Cardiovasc Surg (Torino). 1994;35:151-5.

30. Gaensler EA, Carrington CB. Open biopsy for chronic diffuse infiltrative lung disease: clinical, roentgenographic, and physiological correlations in 502 patients. Ann Thorac Surg. 1980;30:411-26. 